\title{
A Beneficial Interaction between Imipenem and Piperacillin Possibly through Their Renal Excretory Process
}

\author{
Hiroshi SaItoh,,${ }^{* a}$ Masako Oda, ${ }^{a}$ Toru Gyotoku,,${ }^{a}$ Michiya Kobayashi, ${ }^{a}$ Hiroko FuJISAKi, ${ }^{b}$ and \\ Hitoshi SEKIKAWA ${ }^{c}$ \\ ${ }^{a}$ Department of Pharmaceutics, Faculty of Pharmaceutical Sciences, Health Sciences University of Hokkaido; \\ ${ }^{c}$ Department of Biopharmaceutics, Faculty of Pharmaceutical Sciences, Health Sciences University of Hokkaido; 1757 \\ Kanazawa, Ishikari-Tobetsu, Hokkaido 061-0293, Japan: and ${ }^{b}$ Department of Pharmacy, Health Sciences University of \\ Hokkaido Hospital; 2-5-1 Ainosato, Kita-ku, Sapporo 002-8072, Japan.
}

Received March 25, 2006; accepted August 14, 2006

In order to assess the beneficial mechanism of the concomitant use of imipenem (IPM) with piperacillin (PIPC) for the treatment of serious infectious diseases such as sepsis, the effects of PIPC on the uptake of IPM by rat renal cortical slices and on the plasma concentrations of IPM after intravenous infusion to rabbits were studied. The uptake of IPM by the rat renal cortical slices was significantly inhibited by p-aminohippurate, probenecid and PIPC whereas the uptake of PIPC by the slices was slightly decreased in the presence of IPM. When IPM was administered together with PIPC by 1-h infusion, the plasma concentrations of IPM were significantly increased during the infusion. These results imply that PIPC possibly interferes with the renal transport of IPM mediated by an organic anion transporter across the renal basolateral membranes, which leads to a longer period above the minimum inhibitory concentrations of IPM.

Key words imipenem; piperacillin; interaction; renal transport; organic anion transporter

Antibiotics are often coadministered to patients with severe infectious diseases. We previously investigated the actual situation of parenteral antibiotics use at the Hokkaido Prefectural Hospital Haboro and found that a combination therapy of imipenem (IPM) and piperacillin (PIPC) was often undertaken for the treatment of serious infections such as sepsis. In most cases, this treatment was shifted from PIPC monotherapy to combination therapy of IPM and PIPC with clinical deterioration, in spite of the fact that the antimicrobial spectrum widely overlapped in these two $\beta$-lactam antibiotics. The combination therapy of an appropriate $\beta$-lactam antibiotic and an aminoglycoside or a fluoroquinolone is generally recommended for sepsis. ${ }^{1)}$ On the other hand, a recent review has suggested that the combination therapy of an aminoglycoside and a $\beta$-lactam antibiotic is discouraged for sepsis and, in fact, increases the risk of nephrotoxicity. ${ }^{2)}$ Supporting this, our investigation showed that the combination therapy of an aminoglycoside and IPM or PIPC was much less common compared with the combination therapy of IPM and PIPC at the hospital. Moreover, the physicians empirically realized the outcomes of the concomitant use of IPM with PIPC for severe sepsis although they did not know the exact reason. As antibiotics are often expensive, a combination therapy has to be chosen to surpass monotherapy on the basis of potential advantages such as synergism and prevention of developed resistance. However, little information has been available to date on the benefit of the combination therapy of IPM and PIPC.

It is well known that a variety of $\beta$-lactam antibiotics are excreted into urine via the organic anion transporters (OATs) located on the basolateral membranes of proximal tubules. ${ }^{3)}$ Furthermore, it has been reported that PIPC is capable of inhibiting renal transport of several $\beta$-lactam antibiotics possibly mediated by OATs. ${ }^{4,5}$ However, it has been implied that IPM might be a substrate of a renal OAT. ${ }^{6}$ Thus, a likely benefit of combination therapy of IPM and PIPC over IPM or PIPC monotherapy is that PIPC interferes with the renal transport of IPM via an OAT, retards the renal clearance of IPM, and maintains the high blood concentration of IPM. Moreover, as IPM is one of the most nephrotoxic $\beta$-lactam antibiotics, ${ }^{7)}$ it is also likely that the combination therapy of IPM and PIPC is beneficial in reducing this nephrotoxicity. However, there have been no reports demonstrating that IPM and PIPC mutually interfere with their renal transport via an OAT. In order to clarify the clinical benefit of the combination therapy of IPM and PIPC, the pharmacokinetic interactions between these two $\beta$-lactam antibiotics were investigated in this study by measuring their uptake by rat renal cortical slices and by determining blood concentrations after intravenous infusion to rabbits.

\section{MATERIALS AND METHODS}

Materials Imipenem $\left(\right.$ Tienam $\left.^{\circledR}\right)$ and piperacillin sodium $\left(\right.$ Pentcillin ${ }^{\circledR}$ ) were obtained from Banyu Pharmaceutical Co. (Tokyo, Japan) and Toyama Chemical Co. (Tokyo), respectively. $p$-Aminohippurate $(\mathrm{PAH})$ and probenecid were purchased from Wako Pure Chemical Ind. (Osaka, Japan). Other reagents were of the highest grade available.

In Vitro Uptake by Rat Renal Cortical Slices In this study the animal experiments were performed in accordance with the Guidelines for the Care and Use of Laboratory Animals in the Health Sciences University of Hokkaido. Animals were housed for at least 2 weeks before experiments with free access to food and water at $25 \pm 3{ }^{\circ} \mathrm{C}$ and $50 \pm 20 \%$ relative humidity under a 12 -h light/dark cycle, without attrition. For the uptake experiments of IPM and PIPC by rat renal cortical slices, male Wistar rats $(10-12$ weeks, Hokudo, Sapporo, Japan) weighing $300-400 \mathrm{~g}$ were anesthetized by intraperitoneal injection of sodium pentobarbital (40 mg/kg, Dainippon Pharmaceuticals, Osaka) and kidneys were promptly removed, decapsulated and placed in ice-cold saline. Renal cortical slices (approximately $10-20 \mathrm{mg} / \mathrm{slice}$ ) with thickness about $0.5 \mathrm{~mm}$ were cut using a conventional 


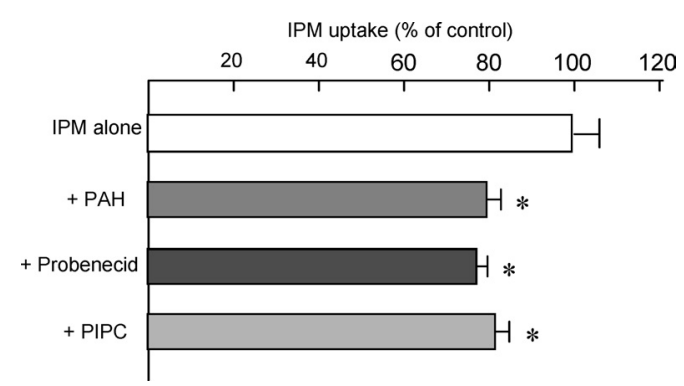

Fig. 1. Effect of PAH, Probenecid, and PIPC on the Uptake of IPM by Rat Renal Cortical Slices

Rat renal cortical slices were incubated in DPBS ( $\mathrm{pH}$ 7.4) including $0.1 \mathrm{~mm}$ IPM in the absence or presence of $5 \mathrm{~mm}$ PAH, probenecid, and PIPC for $30 \mathrm{~min}$ at $37^{\circ} \mathrm{C}$. Each column represents the mean with S.E. of three to six experiments. $* p<0.05$, significantly different from IPM alone (Tukey-Kramer test).

blade. Two cortical slices were prepared from each kidney and washed in Dulbecco's phosphate-buffered saline (DPBS), which consisted of $137 \mathrm{~mm} \mathrm{NaCl}, 3 \mathrm{~mm} \mathrm{KCl}, 8 \mathrm{~mm}$ $\mathrm{Na}_{2} \mathrm{HPO}_{4}, 1.5 \mathrm{~mm} \mathrm{KH} \mathrm{PO}_{4}, 1 \mathrm{~mm} \mathrm{CaCl}, 0.5 \mathrm{~mm} \mathrm{MgCl}$, and $5 \mathrm{mM}$ D-glucose, $\mathrm{pH}$ 7.4). DPBS was oxygenated by $95 \%$ $\mathrm{O}_{2}-5 \% \mathrm{CO}_{2}$ for 30 min before use and $\mathrm{pH}$ was always adjusted to 7.4. Each slice was placed in $3 \mathrm{ml}$ of DPBS containing drugs and incubated at $37{ }^{\circ} \mathrm{C}$ for $30 \mathrm{~min}$. After incubation, the slice was promptly recovered, gently blotted and weighed, then homogenized in a mixture of DPBS and methanol $(1: 1)$ using a polytron. The homogenate obtained was centrifuged at $3000 \times \boldsymbol{g}$ for $10 \mathrm{~min}$ and the supernatant was applied to HPLC assay. A tissue blank was prepared with the drug-free incubation medium. Uptake of IPM was calculated as the S/M ratio (i.e., the concentration of IPM per gram of kidney tissue divided by the concentration of IPM per milliliter of incubation medium).

In Vivo Intravenous Infusion to Rabbits The IPM solution $(3 \mathrm{mg} / \mathrm{ml})$ and the mixture of IPM and PIPC (IPM, 3 $\mathrm{mg} / \mathrm{ml}$; PIPC, $10 \mathrm{mg} / \mathrm{ml}$ ) were prepared by diluting Tienam ${ }^{\circledR}$ and Pentcillin ${ }^{\circledR}$ with saline. For 1-h infusion, a needle was fixed on an ear vein of a conscious male Japanese white rabbit weighing 3-4 kg (Hokudo) and then linked to a microinfusion pump. Ten milliliters of IPM solution or the mixture of IPM and PIPC were injected via the pump. One milliliter of blood was taken from the other side of the ear vein immediately thereafter and at $0.5,1,1.25,1.5,2$, and $3 \mathrm{~h}$ after the start of injection and treated as stated above. It was immediately centrifuged at $3000 \times \boldsymbol{g}$ for $15 \mathrm{~min}$ and the plasma obtained was mixed with the same volume of saline containing $200 \mathrm{~mm}$ 3-morpholinopropanesulfonic acid (MOPS) to prevent the degradation of IPM. The mixture was stored at $-30{ }^{\circ} \mathrm{C}$ until assay. The plasma sample $(0.4 \mathrm{ml})$ was mixed with $0.2 \mathrm{ml}$ of methanol, allowed to stand for $10 \mathrm{~min}$, and then centrifuged at $3000 \times \boldsymbol{g}$ for $15 \mathrm{~min}$. An aliquot of the supernatant was used for HPLC assay. The interval of crossover administration was at least $7 \mathrm{~d}$.

HPLC Analysis The HPLC system consisted of a Shimadzu LC-10AS and a Shimadzu SPD-10A UV-VIS detector. A column of Cosmosil 5C18-AR $(4.6 \times 150 \mathrm{~mm}$, Nacalai Tesque, Kyoto, Japan) was used as a stationary phase. The mobile phases were $10 \mathrm{~mm}$ MOPS-acetonitrile $(100: 5)$ for IPM and $50 \mathrm{mM} \mathrm{KH}_{2} \mathrm{PO}_{4}$-methanol-acetonitrile $(3: 1: 1)$ for PIPC. The flow rates were $0.5 \mathrm{ml} / \mathrm{min}$ for IPM and 1.0 $\mathrm{ml} / \mathrm{min}$ for PIPC, and wavelength for detection of IPM and

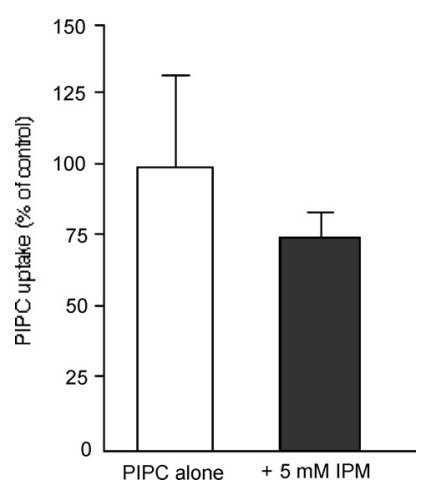

Fig. 2. Effect of IPM on the Uptake of PIPC by Rat Renal Cortical Slices

Rat renal cortical slices were incubated in DPBS (pH 7.4) including $0.1 \mathrm{mM} \mathrm{PIPC}$ in the absence or presence of $5 \mathrm{~mm}$ IPM for $30 \mathrm{~min}$ at $37^{\circ} \mathrm{C}$. Each column represents the mean with S.E. of three experiments.

PIPC was $300 \mathrm{~nm}$ and $220 \mathrm{~nm}$, respectively. Column temperature was set at $30^{\circ} \mathrm{C}$ for IPM and $50^{\circ} \mathrm{C}$ for PIPC, respectively. Under these HPLC conditions IPM and PIPC were eluted within $10 \mathrm{~min}$ and determined with the coefficients of variation less than $7.5 \%$.

Data and Statistical Analysis All data are presented as the means with S.E. from 3 to 5 experiments. Statistical comparisons were performed by Student's $t$-test for two groups and Tukey-Kramer test for multiple groups. $p$ value less than 0.05 was considered to be significant.

\section{RESULTS AND DISCUSSION}

The study using renal cortical slices from animal kidney was devised more than 50 years ago and it is still useful for determining the drug transport in the kidney, ${ }^{8,9)}$ although a recent immunocytochemical study has pointed out several issues of the in vitro method. ${ }^{10)}$ It is assumed that the tubular lumen is substantially collapsed in the rat renal cortical slices and that the uptake in them preferentially reflects the transport from the basolateral side. ${ }^{11)}$ Among several members of the OAT family, OAT1, OAT2 and OAT3 are localized on the basolateral membranes of the middle portion of the proximal tubules ${ }^{12,13)}$ and OAT1 and OAT3 preferentially carry various $\beta$-lactam antibiotics. ${ }^{3)}$ In order to know whether IPM is transported as a substrate of an OAT across the renal basolateral membranes, the effect of two OAT-related compounds, PAH and probenecid, was first examined on the uptake of IPM in the rat renal cortical slices. PAH and probenecid are often used as a prototype substrate of renal OATs and as a potent inhibitor of OATs, respectively. ${ }^{8,14)}$ As shown in Fig. 1, the uptake of IPM $(0.1 \mathrm{mM})$ by rat renal cortical slices during 30-min incubation significantly decreased to $79.5 \pm 2.8 \%$ and $78.0 \pm 2.8 \%$ of IPM alone in the presence of PAH (5 mM) and probenecid $(5 \mathrm{mM})$, respectively. It is assumed that IPM is in part taken up from blood into the proximal tubules, being mediated by an OAT. We further examined the possible interaction between IPM and PIPC in the uptake by rat renal cortical slices. As shown in Fig. 1, PIPC (5 mM) also lowered the uptake of IPM to $82.4 \pm 7.7 \%$ of IPM alone. The magnitude of inhibitory effect of PIPC seemed almost comparable to those obtained with PAH and probenecid. Figure 2 shows the effect of IPM ( $5 \mathrm{mM})$ on the uptake of PIPC $(0.1 \mathrm{mM})$ by rat renal cortical slices. IPM decreased PIPC uptake weakly 


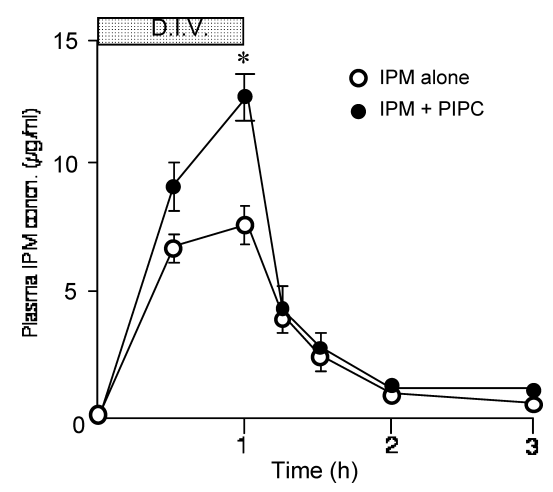

Fig. 3. Plasma Concentration-Time Curves of IPM after Intravenous Infusion to Rabbits with or without PIPC

Ten milliliters of IPM solution $(3 \mathrm{mg} / \mathrm{ml})$ or the mixture of IPM and PIPC (IPM $3 \mathrm{mg} / \mathrm{ml}$; PIPC, $10 \mathrm{mg} / \mathrm{ml}$ ) was injected over $1 \mathrm{~h}$ via a micro-infusion pump. One milliliter of blood was taken from the other side of the ear vein at designated times. Each point represents then mean \pm S.E. of four experiments. $* p<0.05$, significantly different from IPM alone (Student's $t$-test)

but it was not statistically significant. Separately, we investigated the plasma concentrations of IPM and PIPC administered to rats by bolus injection with or without probenecid and found that the decreases in these concentrations of both $\beta$-lactam antibiotics were significantly retarded when administered with probenecid (unpublished data). These results supported that IPM and PIPC are substrates of an OAT. Therefore, it was considered that the less than significant uptake of PIPC in the presence of IPM shown in Fig. 2 is due to wide variety in control values (PIPC alone). Although we cannot conclude which OAT is directly involved in the transport of IPM at this moment, the present results offer the possibility that IPM and PIPC mutually interfere with their transport mediated by an OAT across the renal basolateral membranes in rats.

Based on the above results, it was expected that PIPC interfered with the renal clearance of IPM and elevated the IPM blood concentrations when the two were administered together to patients with serious infectious diseases. As shown in Fig. 1, however, the decrease in IPM uptake by rat renal cortical slices in the presence of $\mathrm{PAH}$, probenecid, and PIPC was at most $20 \%$ of IPM alone, implying that the contribution of an OAT to the renal clearance of IPM was not so remarkable. Thus, a question arose whether PIPC actually modulates the pharmacokinetics of IPM in vivo. To answer this question, we next investigated the effect of PIPC on the plasma concentrations of IPM when administered to rabbits by 1 -h infusion. Figure 3 shows the plasma concentrationtime profiles of IPM when administered by $1-\mathrm{h}$ infusion to rabbits with or without PIPC. In the absence of PIPC, plasma concentrations of IPM reached nearly a steady state at $30 \mathrm{~min}$ after the start of infusion and the concentration was almost the same at $1 \mathrm{~h}$. After the infusion had been completed, the plasma concentrations of IPM sharply declined, indicating rapid elimination of IPM from systemic circulation. When the same amount of IPM was administered concomitantly with PIPC, the plasma concentrations of IPM continuously increased for $1 \mathrm{~h}$ at which time the concentration was significantly greater than that of IPM alone. Following completion of the infusion, no significant differences were observed in the absence or presence of PIPC at sampling times after $1 \mathrm{~h}$.
Table 1. AUC after 1-h Intravenous Infusion of IPM to Rabbits with or without PIPC

\begin{tabular}{lccc}
\hline \hline & \multicolumn{2}{c}{$A U C(\mu \mathrm{g} \cdot \mathrm{h})$} & \\
\cline { 2 - 3 } Time (h) & IPM alone & IPM+PIPC & \\
\hline $0-0.5$ & $1.57 \pm 0.23$ & $2.18 \pm 0.35$ & 139 \\
$0-1$ & $4.94 \pm 0.68$ & $7.40 \pm 0.96$ & 150 \\
$0-1.25$ & $6.30 \pm 0.82$ & $9.40 \pm 1.12$ & 149 \\
$0-1.5$ & $7.03 \pm 0.87$ & $10.2 \pm 1.21$ & 145 \\
$0-2$ & $7.70 \pm 1.00$ & $11.8 \pm 1.64$ & 145 \\
$0-3$ & $8.16 \pm 1.17$ & $11.8 \pm 1.64$ & 145 \\
\hline
\end{tabular}

$A U C$ was calculated by trapezoidal method.

It was considered that the significantly elevated plasma concentrations of IPM during the 1-h infusion were presumably due to the modulation of renal clearance of IPM via an OAT by PIPC. Table 1 shows $A U C$ of IPM calculated from time 0 to each sampling time up to $3 \mathrm{~h}$. The $A U C$ of IPM in the presence of PIPC was always 1.4 to 1.5 -fold greater than those of IPM alone, however, there was no significant difference between them at any sampling times.

The antibacterial activity of $\beta$-lactam antibiotics including carbapenems is known to be dependent on the time above the minimum inhibitory concentrations $(\mathrm{T}>\mathrm{MIC}){ }^{15,16)}$ According to the present results, the concomitant administration of IPM with PIPC is believed to result in a greater T $>$ MIC of IPM, making the antibacterial activity of IPM more beneficial. The combination therapy of IPM and PIPC to patients with infectious diseases was actually undertaken with 2-h infusion at the Hokkaido Prefectural Hospital Haboro. Although we did not determine the blood concentrations of IPM in these patients, the longer infusion time might be beneficial in producing greater IPM antibacterial activity. Most carbapenems exhibit relatively short elimination half-lives after administration. ${ }^{17)}$ If the renal clearance of other carbapenems is efficiently mediated by OAT, PIPC may also be beneficial in modulating such clearance.

In conclusion, our present results have implied that the combination therapy of IPM and PIPC is beneficial for the treatment of serious infectious diseases through a mechanism by which PIPC interferes with an OAT-mediated IPM transport across the renal basolateral membranes and retards the renal clearance of IPM. Further study is required to know whether these results from rats and rabbits are applicable to patients with serious infectious diseases in vivo.

\section{REFERENCES}

1) Bodmann K. F., Chemotherapy (Basel), 51, 227-233 (2005).

2) Paul M., Silbiger I., Grozinsky S., Soares-Weiser K., Leibovici L., Cochrane Database Syst. Rev., CD003344 (2006).

3) Burckhardt B. C., Burckhardt G., Rev. Physiol. Biochem. Pharmacol., 146, 95-158 (2003).

4) Hayashi T., Watanabe Y., Kumano K., Kitayama R., Yasuda T., Saikawa I., Totsuka K., Kumada T., Shimizu K., J. Antibiot., 39, 699712 (1986).

5) Hayashi T., Watanabe Y., Kumano K., Kitayama R., Yasuda T., Saikawa I., Katahira J., Kumada T., Shimizu K., Antimicrob. Agents Chemother, 32, 912-918 (1988).

6) Hirouchi Y., Naganuma H., Kawahara Y., Okada R., Kamiya A., Inui K., Hori R., Jpn. J. Pharmacol., 66, 1-6 (1994).

7) Tune B. M., Pediatr. Nephrol., 11, 768-772 (1997).

8) Nishimura T., Kato Y., Sai Y., Ogihara T., Tsuji A., J. Pharm. Sci., 93, 
$2558-2566$ (2004).

9) Nagai J., Nasu R., Murakami T., Takano M., Eur. J. Pharmacol., 525, $128-134$ (2005).

10) Crljen V., Sabolic I., Susac J., Appenroth D., Herak-Kramberger C. M., Ljubojevec M., Anzai N., Antolovic R., Burckhardt G., Fleck C., Sabolic I., Pflugers Arch-Eur. J. Physiol., 450, 269-279 (2005).

11) Wedeen R. P., Weiner B., Kidney Int., 3, 205-213 (1973).

12) Takeda M., Narikawa S., Hosoyamada M., Cha S. H., Sekine T., Endou H., Eur. J. Pharmacol., 419, 113-120 (2002).

13) Enomoto A., Takeda M., Shimoda M., Narisawa S., Kobayashi Y.-K.,
Kobayashi Y.-S., Yamamoto T., Sekine T., Cha S. H., Niwa T., Endou H., J. Pharmacol. Exp. Ther, 301, 797-802 (2002).

14) Whitley A. C., Sweet D. H., Walle T., Drug Metab. Dispos., 33, 1097-1100 (2005).

15) Burgess D. S., Frei C. R., J. Antimicrob. Chemother, 56, 893-898 (2005).

16) Jaruratanasirikul S., Raungsri N., Punyo J., Sriwiriyajan S., J. Antimicrob. Chemother, 56, 1163-1165 (2005).

17) Mouton J. M., Touw D. J., Horrevorts A. M., Vinks A. A. T. M. M., Clin. Pharmacokinet., 39, 185-201 (2000). 